\title{
Biomonitoreo de la calidad del agua en los ríos ibéricos: lecciones aprendidas
}

\author{
Narcís Prat ${ }^{1, *}$ y Antoni Munné ${ }^{2}$ \\ ${ }^{1}$ Grupo de Investigación F.E.M., Dept. Ecología, Universidad de Barcelona. \\ 2 Agencia Catalana del Agua. \\ * Autor responsable de la correspondencia: nprat@ub.edu
}

Recibido: 06/03/2013 Aceptado: 04/02/2014

\begin{abstract}
Biomonitoring of water quality in Iberian rivers: Learned lessons

A Decalogue summarizing the experience over 30 years in biomonitoring is provided by the authors with two main focuses, the use of macroinvertebrates as biological indicators, and the design of systems to evaluate the ecological status in rivers and streams. After a general introduction and a summary of the origins of biomonitoring in Spain, the authors examine the papers on this topic published in the Limnetica journal. The authors use the data of such papers to illustrate the points that have to be taken into account in order to design biomonitoring programs, and summarized these ideas in a Decalogue. The Decalogue contents all required steps to produce a proper biomonitoring system that will be normative compliant with the specifications of the Water Framework Directive (WFD). The 10 parts of the Decalogue are: 1) how to establish a sampling network; 2) human and material resources available for sampling; 3) definition of the river typologies; 4) how to establish the reference conditions; 5) sampling protocols to be used; 6) biological metrics to be applied; 7) setting the threshold between the different quality classes and its relationship with the pressures; 8) ecological quality maps and output system by using the EQR (Ecological Quality Ratio); 9) quality standards and control; 10) setting the quality objectives and definition of the adequate measures to recover water quality issues. In the Limnetica journal since 1981, a total of 641 papers on aquatic ecology have been published, $42 \%$ were on rivers and $18 \%$ specifically in biomonitoring. Most of the published papers in biomonitoring used macroinvertebrates $(88 \%$ ), while less than $20 \%$ used the reference condition and/or river typology approach to set the quality classes thresholds. However, despite the existence of the WFD since the year 2000, many papers published in Limnetica after 2002 (at this year a Limnetica's special issue on biomonitoring in Mediterranean streams was published), do not met the requirements of this Directive. Today, given the experience gained in the implementation of the WFD any biomonitoring system developed in Europe must be comparable to the existing ones and especially with the common intercalibration metric (ICM).The Decalogue present the necessary steps for a system to be compliant with the WFD regulations.
\end{abstract}

Key words: Biomonitoring, macroinvertebrates, decalogue, water quality, intercalibration, Water Framework Directive.

\section{RESUMEN}

Biomonitoreo de la calidad del agua en los ríos ibéricos: lecciones aprendidas

Los autores resumen en un decálogo la experiencia de más de 30 años de estudio de los ríos españoles con dos focos principales: el uso de macroinvertebrados para la obtención de la calidad biológica y el diseño de sistemas de evaluación del estado ecológico de los ríos. Después de una introducción general y de un resumen de cómo se gestaron los estudios de biomonitoreo en España, los autores analizan los trabajos que se han publicado en la revista Limnetica sobre este tema. Se usan estos datos para ilustrar los puntos relevantes en el diseño y aplicación del biomonitoreo, que es posteriormente resumido en un decálogo. Este está pensado para contener todos los puntos necesarios para que el diseño de un sistema de biomonitoreo cumpla las especificaciones de la Directiva Marco del Agua (DMA). Los diez puntos analizados son: 1) establecimiento de la zona de estudio; 2) recursos humanos y materiales disponibles; 3) tipología de ríos; 4) establecimiento de las condiciones de referencia; 5) protocolos de muestreo; 6) métricas a utilizar; 7) establecimiento de los cortes de calidad y relación de las métricas con las presiones; 8) mapas de estado ecológico usando el valor relativo respecto a los valores de referencia (el EQR: Ecological Quality Ratio); 9) control de calidad; 10) uso de los datos para establecer los objetivos de calidad y el programa de medidas. En la revista Limnetica se han publicado desde su inicio en el año 1981 un total 
de 641 trabajos, de los cuales un $42 \%$ trataban de ríos y un $18 \%$ se centraban exclusivamente en el biomonitoreo. La mayor parte de los trabajos de biomonitoreo publicados utilizaron a los macroinvertebrados como elemento de calidad (88\%), pero relativamente pocos (menos del $20 \%$ ) usaron la tipología y las condiciones de referencia para establecer correctamente las clases de calidad. Se analiza por separado el período anterior al año 2002, y con un número similar de trabajos se advierte un mayor porcentaje de trabajos de biomonitoreo después de 2002. Sin embargo, y a pesar de la existencia de la DMA desde el año 2000, muchos trabajos publicados en Limnetica a partir de 2002 (cuando se hizo un número especial sobre biomonitoreo en ríos mediterráneos) no cumplen los requisitos que demanda esta Directiva. En este momento, después de la experiencia que se tiene por la implementación de la DMA, cualquier sistema de biomonitoreo desarrollado en Europa debe ser comparable a los que existen actualmente, especialmente a la métrica común de intercalibración (ICM).Este decálogo presenta los pasos necesarios para que cuando se diseñe un sistema de muestreo este cumpla los requisitos que demanda la DMA.

Palabras clave: Biomonitoreo, macroinvertebrados, decálogo, calidad del agua, intercalibración, Directiva Marco del Agua.

\section{INTRODUCCIÓN}

Un sistema de biomonitoreo de la calidad del agua pretende diagnosticar el estado de un ecosistema acuático (río, lago, humedal, estuario, etc.) desde un punto de vista integral, usando los organismos que los habitan como principales indicadores de su salud (Woodiwiss, 1964; Margalef, 1969; Bonada et al, 2006). Las comunidades biológicas que habitan los sistemas acuáticos reflejan las condiciones ambientales que se han mantenido en su pasado más reciente, por lo que, mediante su análisis, es posible conocer los efectos que los cambios generados por el hombre tienen sobre los organismos, siendo, en este aspecto, mejores indicadores que los parámetros fisicoquímicos (Hellawell, 1986; Karr \& Chu, 1999). Este concepto ha sido desarrollado desde hace años por diversos autores mediante el uso de diferentes grupos biológicos que habitan los ecosistemas fluviales, como los macroinvertebrados (p. e. Barbour et al., 1995; Chovarec et al., 2000), las algas bentónicas (p. e. Descy, 1979; Kelly et al., 1995; Prygiel et al., 1999), las plantas macrófitas (p. e. Newbold \& Holmes, 1987; Ferreira et al., 2005; Haury et al., 2006), o los peces (p. e. Fausch et al., 1984; Pont et al., 2007). Al igual que en otros países y regiones, en España se han desarrollado diferentes métricas y sistemas de cálculo de la calidad biológica mediante el uso de las comunidades biológicas que habitan nuestros ríos, tanto para los invertebrados bentónicos (p.e. González del Tánago \& García de Jalón, 1984; Prat et al., 1983; Alba-Tercedor,
J. \& Sánchez-Ortega, 1988; Alba-Tercedor et al., 2002; Munné \& Prat, 2009), como para las algas bentónicas (p. e. Sabater et al., 1996; Sabater 2000), las macrófitas acuáticas (Suárez et al., 2005; Moreno et al., 2006), o las comunidades de peces (Sostoa et al., 2004; Benejam et al., 2008).

Tanto la Directiva Marco del Agua (DMA) (2000/60/CE) en Europa (European Commission, 2000), como la Clean Water Act en Estados Unidos han adoptado el uso de índices biológicos para la diagnosis de la calidad de los sistemas acuáticos, lo que ha llevado al desarrollo de protocolos de biomonitoreo estandarizados (Barbour et al., 1999), y a la intercomparación e intercalibración de métodos y umbrales de calidad entre países y regiones (Buffagni \& Furse, 2006; European Commission, 2013). Una revisión del uso de bioindicadores a diferentes niveles de organización y complejidad puede ser consultada en Gerhardt, 1999, y para los macroinvertebrados puede consultarse la revisión de Bonada et al., (2006). Actualmente se han desarrollado y extendido en Europa más de 300 sistemas diferentes de biomonitoreo fruto de la puesta en marcha y aplicación de la Directiva Marco del Agua (Birk et al., 2012), lo que hace necesario estandarizar los requerimientos mínimos que deben cumplir los índices y métricas de los diferentes sistemas para la correcta valoración del estado de los ecosistemas acuáticos. Estos deben poderse comparar, por lo que es necesario una correcta intercalibración de los mismos. El uso del concepto "condiciones de referencia" ha resultado de gran utilidad para la estandarización 
de las métricas, ya que el nivel de calidad de un punto se deriva con respecto al valor de la métrica que se utiliza en un punto exento de presiones antrópicas (estación de referencia) (Bailey et al., 1998), lo que resulta esencial para la comparación de la calidad biológica de los ecosistemas acuáticos entre países, regiones o cuencas.

La calidad del agua se monitorea habitualmente usando parámetros fisicoquímicos, los cuales informan de la situación en el momento de la toma de la muestra, mientras que el biomonitoreo incorpora una dimensión temporal a la medida, ya que si bien la calidad fisicoquímica se recupera inmediatamente, la calidad biológica tarda de semanas a meses, o años, según el organismo utilizado (Rosenberg \& Resh, 1993). Más recientemente, la DMA ha incorporado el uso de la caracterización hidromorfológica como complemento en la diagnosis de la calidad de los ecosistemas acuáticos (Petts, 2008; Poff \& Zimmerman, 2010; Barquín \& Martínez-Capel, 2011). La singularidad del biomonitoreo, con respecto a los tradicionales análisis fisicoquímicos, subyace en que la estructura y composición de la biota es, de manera natural, muy variable entre diferentes cuencas y ecorregiones (más cuanto más alejadas) (Omernik \& Bailey, 1997; Vinson \& Hawkins, 1998; Sponseller et al., 2001), y dentro de una misma cuenca hidrográfica (entre la cabecera y la parte final del río) (Minshall et al., 1985) y, por consiguiente, es preciso un profundo conocimiento de la comunidad propia de cada sistema antes de efectuar una diagnosis o aplicar los índices biológicos (Armitage et al., 1990; Heiskanen et al., 2004). Estos aspectos han sido abordados de forma detallada por la implementación de la DMA con una serie de manuales, que intentan clarificar y uniformizar la manera en que los diferentes Estados de la UE deben abordar el problema (Wallin et al., 2003).

Más recientemente, se han incorporado al biomonitoreo métricas funcionales, calculadas a partir de la actividad metabólica de los organismos, o cambios en su fisiología, morfometría, comportamiento, etc. Un ejemplo son los biomarcadores, (desde enzimas simples a marcadores genéticos) que completan la información dada por las métricas tradicionales (p. e. Barata et al.,
2004). Los biomarcadores no tienen, por el momento, un uso generalizado entre las administraciones del agua, y se aplican tan solo en algunos casos para la determinación de los efectos subletales producidos por la contaminación latente de baja intensidad (p. e. Quirós et al., 2007; Prat et al., 2013), o cuando se quieren investigar las causas concretas de la degradación de la comunidad (p. e. Damasio et al., 2007).

La solución al problema de cómo muestrear y comparar los datos ha sido objeto de innumerables estudios en Estados Unidos, Australia o Europa. En Europa, la Directiva Marco del Agua (DMA) ha obligado a buscar un mecanismo para poder comparar los datos entre países con sistemas muy diferentes de medida del estado de los ríos (Buffagni \& Furse, 2006). Ello se ha concretado en protocolos de estudio y en documentos de normalización que incluyen normas CEN (Comité Europeo de Normalización) -o AENOR, en el caso de España- o diversas publicadas en su Diario Oficial (p. e. European Commission, 2008). En España, la revista Limnetica fue pionera en la publicación de un protocolo estandarizado para los ríos mediterráneos españoles (el protocolo Guadalmed: Prat, 2002). El presente artículo pretende realizar una revisión de la evolución de la aplicación de los índices de calidad biológica en los ríos ibéricos, basada en el análisis de las publicaciones editadas en Limnetica antes y después de la publicación de Directiva Marco del Agua. Finalmente los autores utilizan un formato a modo de decálogo para enumerar los pasos necesarios a fin de establecer un buen programa de biomonitoreo que permita una buena interfaz entre los avances científicos y las necesidades de los gestores.

\section{RESULTADOS}

\section{El biomonitoreo en la revista Limnetica}

En España, en el año 1982, se diseñó el primer índice biológico para los ríos Besòs y Llobregat en Cataluña (Prat et al., 1983). En Limnetica se publicó a mediados de la década de 1980 uno de los primeros trabajos sobre el tema (Gon- 


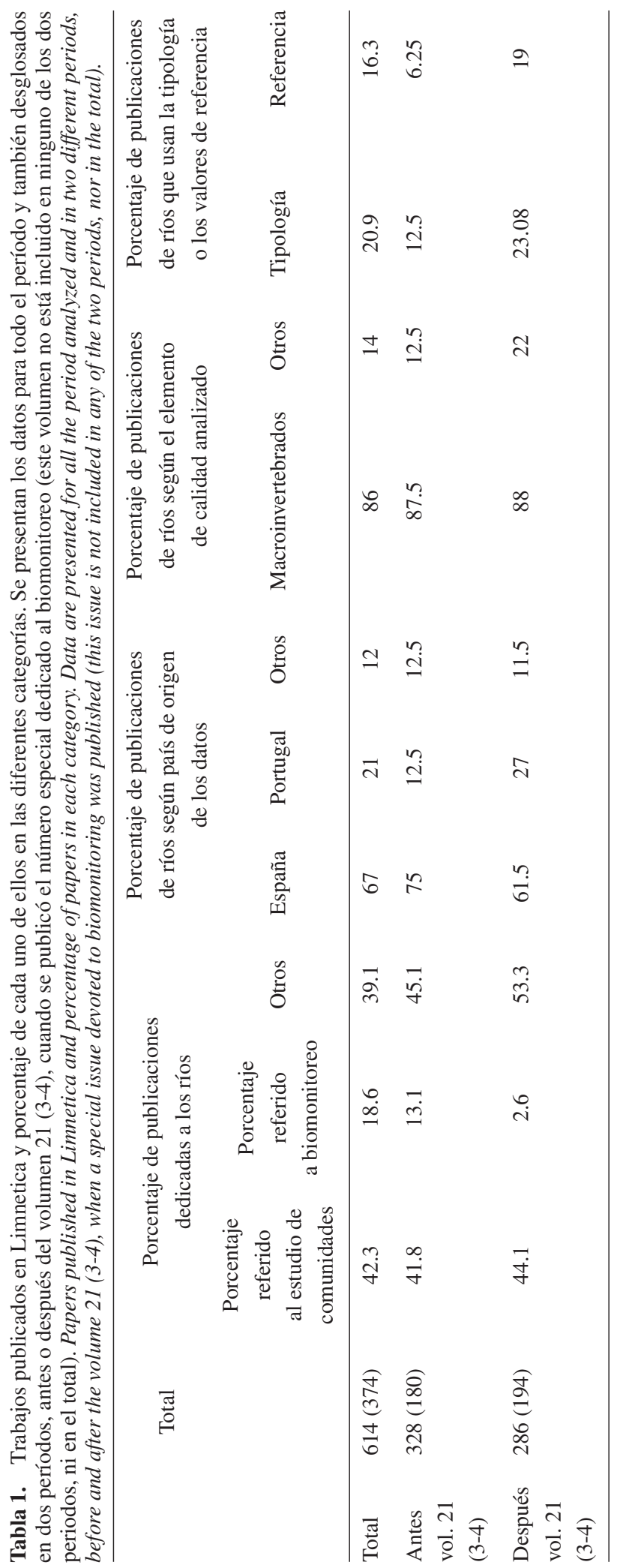


zález del Tánago \& García de Jalón, 1984) centrado en la adaptación de los índices de calidad mediante el uso de macroinvertebrados en el río Jarama. A partir de trabajos anteriores, el profesor Alba-Tercedor adaptó el índice BMWP, previamente desarrollado en Inglaterra (Hellawell, 1986), a los ríos españoles (el BMWP'), que fue también publicado en Limnetica (Alba-Tercedor \& Sánchez-Ortega, 1988), trabajo que ha sido repetidamente citado y se ha convertido en el índice biológico de referencia y más ampliamente usado en España en los últimos años. La historia de la bioindicación hasta la década de 1990 , con su correspondiente bibliografía, puede consultarse en Alba-Tercedor \& Prat, (1992). Posteriormente el índice BMWP' fue revisado y ampliado para dar cobertura al conjunto de los ríos ibéricos (el IBMWP), también publicado en Limnetica (Alba-Tercedor et al, 2002).

Podemos obtener una pequeña idea del interés que ha despertado este tema en España examinando los trabajos publicados en la revista Limnetica desde 1984 (Tabla 1). Los resultados globales no incluyen algunos números especiales dedicados a lagos. Desde 1984 se han publicado en Limnetica un total de 614 trabajos, de los cuales 263 tratan específicamente de ríos (42\%), y de estos, 49 se centran en la aplicación de índices de calidad y biomonitoreo (18.3\%). El biomonitoreo ha tenido presencia en la revista Limnetica ya desde los inicios de la aplicación de sistemas de biomonitoreo en la península Ibérica (en 1984), con un promedio aproximado de 1 trabajo por volumen publicado. En todo este período destaca la edición especial de Limnetica (volumen 21 (3-4) dedicado a los resultados obtenidos del proyecto GUADALMED. Este fue desarrollado entre los años 1998 y 2002, y en él se llevó a cabo un ejercicio de aplicación de estos índices en los ríos de la vertiente mediterránea de la península Ibérica y las islas Baleares siguiendo las directrices expuestas por la DMA (Prat, 2002). En la Tabla 1 puede observarse que los trabajos de biomonitoreo casi se duplican en el período posterior a la publicación de este monográfico. Las aportaciones vienen tanto de investigadores españoles como portugueses, cuya importancia crece en la etapa poste- rior al año 2002. Las conclusiones y datos del proyecto GUADALMED han sido usados por diversas administraciones del agua en España para el proceso de implementación de la DMA (p. e. Oscoz et al., 2007; Munné et al., 2012) y para la intercalibración con diversos sistemas de biomonitoreo empleados en Europa (Munné \& Prat, 2009; Feio et al., 2013). Su metodología y resultados permitieron el desarrollo de la Instrucción Técnica de Planificación Hidrológica (IPH) elaborada por el ministerio de Medio Ambiente a finales de 2008 en España (MARM, 2008).

Una vez finalizados el primer y segundo ejercicios de intercalibración de indicadores biológicos a nivel europeo (ver: http://ec.europa.eu/ environment/water/water-framework/objectives/ status_en.htm) (Birk et al., 2012), y fruto de la experiencia adquirida en este terreno en los últimos años por los dos autores, el presente trabajo pretende sintetizar los elementos clave para diseñar un correcto sistema de evaluación de la calidad del agua mediante el uso de organismos acuáticos (Tabla 2). Aunque la experiencia de los autores del trabajo se centra fundamentalmente en el uso de macroinvertebrados en ríos, los resultados y pasos expuestos podrían también aplicarse al resto de elementos biológicos propuestos por la DMA para la diagnosis del estado de las masas de agua superficial.

\section{Decálogo para el diseño y uso de un sistema de evaluación de la calidad biológica de un río}

\section{Establecimiento de la zona de estudio}

Los procesos y cambios que se producen a lo largo de los cursos fluviales pueden ser más fácilmente interpretados si se posee una correcta información a nivel de cuenca (p. e. Tiner, 2004). Por ello las estaciones de muestreo deben situarse estratégicamente de manera que aporten información relevante del efecto de las principales presiones que alteran o pueden alterar el estado de los cursos fluviales, pero deben también tenerse en cuenta los cambios fisiográficos e hidromorfológicos naturales a lo largo de los ríos para ajustar dónde se sitúan los puntos de muestreo (Leeks et al., 1997). La adecuada situación de las esta- 
ciones de muestreo es esencial para discriminar posibles variaciones en la composición y abundancia de las comunidades biológicas debidos a condiciones naturales del cauce (Vila-Gispert et al., 2002).

De forma habitual, se aconseja que las estaciones estén separadas entre ellas entre 1 y $5 \mathrm{~km}$, aunque este extremo es muy variable en función de la cuenca y de los objetivos del monitoreo. Asimismo, hay que asegurarse de que los vertidos que pueden provocar cambios importantes en la calidad del agua se hayan mezclado perfectamente con las aguas del río, por ello la estación aguas abajo del vertido debe estar a una distancia prudencial (en función de caudal del río entre 100 metros y 1 kilómetro). También se debe tener en cuenta la facilidad de acceso al punto de toma de la muestra, la duración del muestreo y los recorridos entre estaciones para optimizar el tiempo. El número total de estaciones se fijará en función de los recursos económicos y humanos disponibles (ver punto 2 de la Tabla 2).

\section{Recursos humanos y materiales}

La aplicación del protocolo de muestreo, y posteriormente el procesado de las muestras e identificación de taxones, requiere de personal especializado y conocedor de los métodos a aplicar. Una mala praxis en la recogida de muestras, o errores en el proceso de tratamiento de las mismas, puede comportar la mala clasificación del estado final de las masas de agua o tramos de río a estudiar (Munné et al., 2003). En general, para un estudio de biomonitoreo pueden seguirse dos estrategias: 1) Realizar los estudios y seguimiento con el personal propio de la organización encargada del monitoreo, aunque la complejidad de usar varios elementos biológicos de manera combinada (diatomeas, macrófitos, macroinvertebrados, peces, etc.) dificulta enormemente la internalización de los trabajos por parte de los organismos de cuenca, que por lo general no poseen personal especializado en los conocimientos taxonómicos requeridos. 2) Encargar el monitoreo a través de asistencias técnicas externas, que suele ser la estrategia elegida por la mayoría de las autoridades de cuenca. Se recomienda establecer un sistema de control de calidad ajeno a la propia contratación que permita a los receptores del estudio la confianza de que tanto los muestreos como la identificación taxonómica se han realizado de manera correcta.

En muchos casos, la situación es intermedia, parte de los trabajos, y muy especialmente la dirección y el control del proceso y la redacción de informes, es realizado por la propia administración, y parte por elementos externos, especialmente el trabajo de campo, el procesado de muestras y la identificación de taxones. Casi siempre es necesaria una fase previa para adecuar los protocolos a la realidad de los sistemas estudiados. En la península Ibérica existen ya diferentes herramientas elaboradas tanto por grupos de investigación como por los propios organismos reguladores (p. e. la Dirección General del Agua en España, o el Instituto del Agua en Portugal). En España, por ejemplo, la base de datos de Taxagua (http://www.magrama.gob.es/es/ agua/temas/estado-y-calidad-de-las-aguas/aguassuperficiales/programas-seguimiento/taxagua.aspx), la publicación de claves de identificación taxonómica (p e. www.chebro.es), o las herramientas informáticas para el cálculo de métricas (p. e. http: //www.aca-web.gencat.cat) han sido y serán importantes herramientas cuyo conocimiento previo es clave para la adecuada aplicación de los índices biológicos de calidad. La mejor manera de asegurar que el equipo de trabajo es el adecuado pasa por establecer un sistema de certificación y verificación que permita constatar de antemano, y a posteriori, la profesionalidad del equipo.

\section{Tipología}

Los estudios de tipología de los ríos tienen una larga tradición en ecología (Illies \& Botosaneanu, 1963). Distinguir los diferentes tramos por sus características hidromorfológicas o biológicas ha sido un objetivo recurrente de muchos de los estudios realizados (p.e. Frissell et al., 1986; Ferréol et al., 2005). Para el caso del biomonitoreo, la comparación entre ríos de características hidromorfológicas diferentes requiere establecer tipologías para poder realizar comparaciones (Dodkins et al., 2005). Las diferencias 
en la estructura y la composición de las comunidades biológicas que habitan los sistemas fluviales han sido demostradas en diversos estudios (p.ej.Vinson \& Hawkins, 1998), y en algunos casos pueden tener un fuerte componente biogeográfico, como es el caso de los peces (VilaGispert et al., 2002.

La tipificación de los ríos como herramienta de diagnosis ha sido reconocida en la legislación mas reciente como un elemento previo a todo análisis (p. e. el Anexo II de la DMA). Entre los primeros trabajos de tipología de ríos en España, realizados para ser utilizados en la implementación de la DMA, destacan los realiza- dos en la cuenca del Ebro (Munné \& Prat, 1998), y posteriormente en las cuencas internas de Cataluña (Munné \& Prat, 2002). Estos trabajos se fundamentaban en el análisis de parámetros fisiográficos y ambientales, no alterados por la actividad humana, que repercutían o tenían influencia en la composición taxonómica de las comunidades biológicas tal como demanda la DMA. A partir del proyecto Guadalmed (Prat et al., 2002) se desarrollaron diversos trabajos a partir de parámetros hidromorfológicos y fisiográficos, con una metodología propia para la definición de tipos fluviales, junto al análisis de la composición de las comunidades (Bonada et al., 2002a), me-

Tabla 2. Decálogo para el diseño de un sistema de evaluación de la calidad biológica de un río. Decalogue used to design a system for the evaluation of the biological quality of a stream.

1. Zona de estudio. Zona en la que se quiere realizar la evaluación, habitualmente debería ser una cuenca hidrográfica entera con sus afluentes. La densidad de estaciones de muestreo e intensidad de muestreo debe definirse en función de las alteraciones antrópicas presentes.

2. Recursos humanos y materiales. El alcance del sistema de evaluación debe hacerse de acuerdo con los recursos humanos y materiales disponibles. La formación de los profesionales que van a realizar el estudio es clave para garantizar la calidad de los datos y la fiabilidad de la diagnosis. Hay que asegurarse que se aplica el protocolo de muestreo correctamente, y con posterioridad los datos deben ser procesados, y los taxones identificados correctamente.

3. Tipología. La fauna de macroinvertebrados es muy diferente según la sección del río estudiada, y solo se pueden comparar los ríos que tengan faunas similares, por lo que es preciso establecer una tipología de los ríos, de modo que la evaluación del estado de los mismos se realice para cada tipo de forma independiente.

4. Condiciones de referencia. Se trata de definir una imagen objetivo para cada uno de los tipos que hayamos definido. Es preciso conocer cuáles son las comunidades que existen en los lugares de una tipología que no tenga perturbaciones antrópicas importantes.

5. Protocolos de muestreo. Para la obtención de los datos en el campo se necesita un protocolo de muestreo estandarizado que debe ser adecuado a las métricas que se van a utilizar para establecer el estado ecológico. No solo el método de muestreo es objeto del protocolo, sino también la manera de tratar las muestras en el laboratorio y su conservación.

6. Métricas. Una métrica sintetiza en un valor la condición biológica del mismo a partir de los datos de presencia y abundancia de los organismos capturados. Qué métricas usar, y cómo validarlas, debe ser objeto también del protocolo.

7. Establecimiento de clases de calidad. Una vez tenemos la o las métricas calculadas, deben distinguirse las diferentes clases de calidad. De forma habitual se distinguen 5 clases: muy buen estado, buen estado, regular o mediocre, malo y pésimo o muy malo. La distinción entre clases debe hacerse de acuerdo con la o las presiones que van a ser objeto de estudio, pudiendo ser una presión concreta (por ejemplo la eutrofización), o bien una más general (degradación general del sistema).

8. Elaboración de mapas de estado ecológico. Establecidos los umbrales de las diferentes clases de calidad, debemos comparar, para cada tipo, los valores obtenidos en el muestreo para cada métrica seleccionada con los valores de referencia, con lo que obtendremos el Ratio de Calidad Ecológica (RCE o EQR por sus siglas en inglés). Con este valor podremos elaborar el mapa del estado, ya que será posible clasificar cada valor dentro de las diferentes categorías de calidad previamente establecidas independientemente del tipo de río.

9. Control de calidad. El proceso debe incluir controles de calidad en las diferentes fases para asegurar que los datos obtenidos son fehacientes y reflejan los valores de calidad con un nivel de certeza aceptable. Hay que controlar todo el proceso, desde la toma de muestras, su conservación, el procesado, la identificación de taxones, el recuento, la aplicación de las métricas e índices de calidad y la obtención final de la diagnosis.

10. Objetivos ambientales. A partir de los mapas podremos definir cuáles son nuestros objetivos de calidad para el futuro. En el caso de que los ríos estén en muy buen estado o en buen estado, el objetivo deberá ser mantenerlo evitando su degradación; en caso de que el estado sea inferior a bueno habrá que investigar las causas de ello (los datos fisicoquímicos y de características del hábitat y las riberas serán determinantes) y establecer las medidas necesarias para que el estado del río mejore en el plazo mínimo de tiempo posible. 
todología que fue utilizada posteriormente por el CEDEX para establecer la tipología que actualmente se usa oficialmente en España para los propósitos de la DMA (MARM, 2008). Por otra parte se han desarrollado también algunos sistemas para establecer tipos fluviales a través de modelos predictivos que usan las comunidades biológicas tanto en los ríos españoles (MEDPACS, Poquet et al., 2009), como en los portugueses (Feio et al., 2009), o a nivel ibérico (Feio \& Poquet, 2011). En ambos casos, como lo que queremos es establecer el estado de los ríos y hay que conocer sus condiciones de referencia, la tipología debe realizarse usando parámetros que no estén alterados por la acción del hombre (Bonada et al., 2002a; Munné \& Prat, 2004).

Algunos trabajos han comparado las tipologías hidromorfológicas con las biológicas tanto en España (Sanchez-Montoya et al., 2007), como en Portugal (Chaves et al., 2011), y han puesto de manifiesto las diferencias entre ellas. Cabe destacar que no siempre las tipologías elaboradas a partir de datos ambientales coinciden exactamente con las agregaciones taxonómicas. Como las tipologías dependen en gran parte de la disponibilidad de datos, cuando la disponibilidad de estos es escasa hay que recurrir a metodologías mixtas que permitan establecer correlaciones entre las comunidades biológicas y las principales características

En el caso de los trabajos publicados en la revista Limnetica (Tabla 1), se ve claramente cómo este aspecto gana importancia después del año 2002 (se doblan los trabajos donde la tipología se utiliza para el establecimiento de diferentes umbrales de calidad). De todas formas, llama la atención ver no se usan tipos de ríos en diversos trabajos publicados en Limnetica recientemente, ni siquiera los oficiales establecidos por el CEDEX (MARM, 2008).

\section{Condiciones de referencia}

El establecimiento de tipos lleva aparejado, de forma inseparable, el buscar para cada uno de ellos la imagen objetivo, cuáles serían las comunidades de estos ríos en ausencia de perturbaciones antrópicas: las condiciones de referencia
(Bailey et al., 1998). Para conocer esta imagen objetivo pueden utilizarse diferentes metodologías. Lo ideal es poder escoger, entre varios lugares del tipo de río a estudiar, tramos que no tengan presiones antrópicas para poder muestrear sus comunidades y establecer, de esta manera, la condición de referencia (Warry \& Hanau, 1993). El método funciona si hay tramos del tipo poco alterados, pero dado que en muchos países y cuencas la actividad humana es importante, no siempre es posible encontrar lugares de referencia, por lo que habrá que utilizar algún método alternativo. En este sentido, la Comisión Europea estableció cuales deberían ser las metodologías a utilizar según la posibilidad o no de que existieran datos históricos o estaciones de referencia (Wallin et al., 2003, Nijboer et al., 2004).

En España existe una considerable bibliografía sobre cómo definir las condiciones de referencia en los ríos, empezando por el proyecto Guadalmed (Bonada et al., 2002c), donde se estableció un protocolo relativamente simple dada la dificultad de obtener algunos datos, mejorado en los trabajos realizados en las cuencas internas de Catalunya, donde se tenían datos más completos de las presiones existentes (Munné \& Prat, 2002,). La metodología Guadalmed se ha implementado especialmente en España y Portugal (Chaves et al., 2006). Particularmente importante ha sido el proceso de intercalibración europea, donde se diseñó una metodología propia para poder establecer condiciones de referencia comunes que permitieran comparar los datos de los ríos europeos mediterráneos (Feio et al., 2013). Asimismo, se han establecido sistemas adecuados para establecer condiciones de referencia en ríos temporales (Sánchez-Montoya et al., 2009).

Los valores de referencia para los diferentes tipos de ríos españoles (para macroinvertebrados y algas por lo menos) se han establecido también oficialmente en España (MARM, 2008). Hoy en día debería ser inhabitual publicar un trabajo donde se presenten datos de ríos de diferente tipo que no se comparen mediante su valor estandarizado, o valor EQR (Ecological Quality Ratio), que resulta de dividir el valor obtenido de cualquier métrica por el valor de referencia de la misma en el tipo de río que se está analizando. 
El uso del valor estandarizado EQR se incrementó en los trabajos presentados en Limnetica después de 2002, donde el número de aquellos que utilizan las condiciones de referencia se triplica, aunque estos solo suponen el $20 \%$ de los trabajos publicados (Tabla 1).

\section{Protocolos de muestreo}

La comparación de muestras (entre sí, o respecto a una referencia) para establecer la calidad biológica requiere que estas hayan sido tomadas de la misma forma. Como las redes de monitoreo pueden ser muy amplias, y no todas las muestras van a ser tomadas por las mismas personas o equipos de muestreo, es importante establecer, de forma muy clara, un protocolo de cómo muestrear y de cómo procesar las muestras para cada uno de los elementos de calidad a muestrear. El protocolo debe ser lo más detallado posible, incluyendo las características de los aparatos de toma de muestras (tipos de redes, luz de la malla, etc.), y como estos deben usarse en el campo (Hering et al., 2006). El número de unidades de muestreo y cómo ubicarlas en el lecho heterogéneo del río deben expresarse de forma clara. La forma en que se obtienen los organismos en función del sustrato y las condiciones hidráulicas del río condicionan el resultado del muestreo (Stark, 1993). Asimismo, cómo filtrar y fijar las muestras en el campo, y cómo tratarlas en el laboratorio, debe ser claramente explicado. El número de organismos que hay que contar y cómo hacerlo es clave para los resultados finales (Doberstein et al., 2000). Una comparación entre diversos métodos y protocolos de muestreo usados en Europa puede encontrarse en Friberg et al., (2006).

El uso de datos cuantitativos (expresados como individuos/metro cuadrado), cualitativos (presencia o ausencia) o semicuantitativos (p.e. porcentajes o clases de abundancia), ha generado algunos debates. En realidad todos los métodos son igual de buenos, o de malos, en función de su uso. Así, los protocolos basados en datos semicuantitativos, como el protocolo GUADALMED (válido para sistemas que usen índices tipo BMWP) (Jaimez-Cuéllar et al., 2002), son equiparables en su funcionamiento a los cuantitativos
(Munné \& Prat, 2009). De todas formas, y dado que la DMA aboga por el uso de métricas de tipo cuantitativo (uso de la densidad de organismos), se han desarrollado para España diferentes metodologías con índices multimétricos que requieren datos cuantitativos (Munné \& Prat, 2009).

Los trabajos de la revista Limnetica sobre biomonitoreo usan casi siempre macroinvertebrados como organismos indicadores (Tabla 1), tanto de forma general como en los dos períodos diferenciados (antes y después de 2002). En los anteriores al año 2002, no se cita ningún protocolo en concreto (excepto en un caso que es un estudio hecho con el sistema RIVPACS). Después de 2002, la mitad de los trabajos citan el protocolo Guadalmed, y usan tanto los macroinvertebrados como los otros indicadores de este protocolo (IHF, QBR, etc.), y esto tanto para estudios extensivos como intensivos, y tanto para España como para Portugal.

\section{Métricas a utilizar}

Los diferentes tipos de métricas a usar, las ventajas e inconvenientes de cada una de ellas, y el uso que se ha hecho en diferentes países, ha sido revisado en diversas ocasiones (Bonada et al., 2006). Para cada una de las métricas debe calcularse no solo el valor absoluto de la misma, sino su valor respecto al valor de referencia (EQR). El uso de índices multimétricos y su comparación con otros índices puede observarse en Mondy et al., 2012.

Una adaptación para ríos mediterráneos y el diseño de un índice multimétrico con base a datos cualitativos, otro con base a datos cuantitativos y la comparación entre ambos puede consultarse en Munné \& Prat, (2009), donde se demuestra que ambos son igual de eficientes frente a la detección del grado de alteración de los ríos analizados (ríos mediterráneos españoles). Tanto los índices unimétricos (p.e. FBILL, IBMWP), como los multimétricos (Munné \& Prat, 2009, 2011), muestran también un comportamiento similar, aunque los multimétricos se correlacionan mejor con el gradiente de presiones (Munne \& Prat, 2009). En España se ha desarrollado la aplicación HIBIM, que permite calcular 46 métricas e índices de calidad, tanto de cualitativas como 
cuantitativas, usando macroinvertebrados, algas diatomeas, macrófitas, peces y los índices de hábitat y bosque de ribera IHF y QBR. Asimismo, a partir de la propia aplicación se pueden generar los valores de las métricas en condiciones de referencia (si se tienen un mínimo de 6 estaciones de referencia) y así calcular los valores EQR.

En el caso de los trabajos publicados en Limnetica, casi todos ellos utilizan el índice IBMWP (a partir del momento en que se generó), aunque muchos de ellos ignoran (incluso después de 2002) los valores y umbrales de calidad específicos de cada tipología (Alba-Tercedor et al., 2002). Es importante resaltar en este punto la existencia de un proceso de intercalibración europea por el cual las métricas usadas en un país se comparan con las propias de otros países, y se utiliza la métrica de intercalibración europea ICM-Star como patrón de todas las demás (Feio et al., 2013).

\section{Establecimiento de las clases de calidad}

Cómo utilizar las métricas calculadas para establecer la calidad, y especialmente cómo se definen los límites entre clases, es también un paso importante en el proceso de elaboración de un protocolo de evaluación de la calidad biológica. Para seleccionar qué métrica vamos a usar, hay que estar seguro de que esta es sensible al gradiente de presiones que queremos analizar o monitorizar tanto a escala de punto de muestreo, como de segmento fluvial, o de toda la cuenca (Munné \& Prat, 2009). El gradiente puede ser específico (por ejemplo la eutrofización, la acidificación o las alteraciones morfológicas), o bien más general (degradación general del ecosistema). Solo en ecosistemas poco alterados es posible diferenciar bien las presiones, por lo que en estos casos se pueden emplear métricas ajustadas a dichas presiones, que las reflejen de forma clara, por ejemplo la presencia de pequeñas presas o una eutrofización no asociada a contaminación orgánica (Benejam et al., 2010). Se recomienda combinar diversas métricas o índices sensibles a diferentes presiones (índices mutimétricos), que proporcionan una mejor diagnosis del estado de los ecosistemas.
Una vez definida la presión hay que correlacionar las métricas con esta y seleccionar, para la elaboración del sistema de medida del estado ecológico, solo aquellas que muestran elevados y significativos coeficientes de correlación con las presiones. Luego hay que determinar si las diversas métricas están muy correlacionadas entre sí para evitar redundancia entre ellas. Con las métricas resultantes y/o la combinación de estas se definen posteriormente los límites o umbrales de calidad, lo que se conoce como las clases de calidad (cinco en la DMA: muy bueno, bueno, moderado, deficiente y malo). Las clases de calidad pueden establecerse de manera equitativa entre ellas solo cuando el gradiente de presión muestra una relación lineal con la métrica que la mide (European Commission, 2005, 2007), si esta condición no se cumple, entonces los umbrales de calidad deberán responder a los criterios concretos que queramos reflejar con dichas clases (Munné \& Prat, 2009).

Como ya se ha comentado en las condiciones de referencia, la valoración del estado de las masas de agua se establece mediante el valor EQR (valor relativo al estado de referencia). La combinación entre métricas en un índice multimétrico debe realizarse mediante la combinación de sus valores EQR, para estandarizar las posibles diferencias en los rangos de variabilidad de las métricas usadas o combinadas. Esto implica que previamente, para cada métrica, sea necesario establecer el valor de referencia para cada tipo, y se hayan calculado los valores EQR. Tal y como establece la DMA, para definir los límites o umbrales de calidad deben calcularse primero los valores de las métricas para el conjunto de sitios de referencia, valores que lógicamente tendrán cierta variabilidad. Para fijar el límite entre bueno y muy bueno y tener en cuenta dicha variabilidad, las guías editadas por la Comisión Europea (European Commission, 2005, 2007) proponen usar el percentil 25 como umbral entre el muy buen estado y el buen estado (calculado este percentil para un mínimo de 6 muestras). Si la métrica seleccionada tiene relación lineal con las presiones, los restantes cortes de calidad se realizan dividiendo en cuatro partes el intervalo entre el valor del límite del muy bueno y el valor mínimo 
de la métrica. Si la relación no es lineal (como ocurre a veces con el índice IBMWP) (Munné \& Prat, 2009), deben buscarse entonces los criterios de corte de las clases de calidad adecuado al tipo de relación entre las métricas y el gradiente de presiones (Alba-Tercedor et al., 2002).

Excepto en los trabajos del volumen especial de Limnetica dedicados al proyecto GUADALMED (Prat, 2002), en los trabajos dedicados al biomonitoreo publicados no hay ninguna referencia a cómo se han establecido las clases de calidad. En muchos casos se utilizan los cortes que publicaron Alba-Tercedor y Sánchez-Ortega en 1988 (sin tener en cuenta ni tipos ni referencias), en otros pocos se fijan unos límites sin explicar el porqué, y en los más recientes sí se adopta la comparación con los valores de referencia, y se proponen los datos que la IPH proporciona para los diferentes tipos de ríos españoles (MARM, 2008), sin realizar un estudio que demuestre la relación de los valores de las métricas con el gradiente de presiones.

\section{Elaboración de mapas de estado ecológico}

Una vez realizado el proceso anterior de cálculo de las métricas, y establecidos los límites entre clases, ya podemos elaborar el mapa de estado ecológico. En este punto hay que combinar muchas veces las métricas biológicas con otras métricas que nos indiquen el estado del sistema desde otro punto de vista, sean las condiciones fisicoquímicas o las condiciones hidromorfológicas. La combinación de los diferentes tipos de métricas puede ser importante para mejorar la estima de la calidad global del sistema siguiendo o no las recomendaciones de la DMA, donde la calidad biológica es más importante que la fisicoquímica o la hidromorfológica. Aunque un hábitat poco adecuado o un bosque de ribera alterado normalmente se reflejan en la composición y estructura de la comunidad de macroinvertebrados, esto puede no ser así cuando el impacto es moderado. Por ello, el combinar indicadores es interesante para tener una mejor perspectiva de la calidad del sistema.

En España existen diversos índices adecuados para la caracterización rápida de la calidad del bosque de ribera (el QBR; Munné et al., 2003) o para el hábitat fluvial (índice IHF) (Prat et al., 2009). Índices usados oficialmente en España en la IPH (MARM, 2008). Un volumen reciente de la revista Limnetica hizo una síntesis de los diferentes índices hidromorfológicos usados en España y cómo se aplican (Barquin \& MartínezCapel, 2011). La CHE (2013) ha publicado una síntesis de los índices hidromorfológicos que está usando en su red de calidad.

\section{Controles de calidad}

Como el objetivo del estudio es elaborar un mapa de calidad, y que este sea público para que los ciudadanos lo conozcan, la publicación del mapa será siempre un momento muy importante e incluso delicado para los gestores. Si en alguna parte del proceso este no se ha realizado correctamente, los valores finales pueden ser inferiores o superiores al valor real y alterar la visión real del estado de las masas de agua. El gestor, cuando publica el mapa, debe tener muy claro que los valores hayan sido contrastados y verificados. Para ello, todo el proceso, desde el diseño del protocolo hasta el mapa final, debe tener un control de calidad. El cumplimiento de normas ISO (o similares), cuando las hubiera, es una primera garantía, pero en algunos casos las normas no existen o no son suficientemente ajustadas al proceso. Como el muestreo y elaboración de los datos es la fase más delicada, hay que extremar los controles en este aspecto. Todos estos procesos deberían estandarizarse e integrarse en los procesos de biomonitoreo que realizan los organismos oficiales.

\section{Objetivos de calidad}

La elaboración de un mapa de calidad no significa el fin del proceso sino su inicio. El obtener un mapa de estado de salud tiene como consecuencia la elaboración de un Programa de Medidas para preservar los ríos que tienen buen o muy buen estado, y restaurar la salud de los que lo tienen inferior a bueno. El Programa de Medidas debe elaborarse con todos los actores implicados, y para cada tramo de río en estado inferior a bueno hay que diseñar las medidas necesarias para su 
recuperación (se deben conocer pues las causas de la degradación), y establecer los objetivos de calidad dentro de un período temporal concreto (Plan Hidrológico o Plan de Gestión de la Demarcación). En Europa, la DMA establece que el proceso dura seis años, desde la implementación de las medidas hasta que se elabora un nuevo mapa de estado para ver la efectividad de las mismas (ciclos de planificación hidrológica).

El programa de medidas tiene un coste económico y social, por lo que requiere un amplio consenso para llevarlo a cabo. Esto implica la participación de los ciudadanos que habitan la cuenca, no solo los del tramo concreto, pues los tramos bajos no se pueden recuperar sin las acciones necesarias aguas arriba. En función de las disponibilidades económicas y de la problemática social se pueden diseñar objetivos concretos para tramos determinados de ríos, o establecer prórrogas o derogaciones temporales de los objetivos establecidos. En la evaluación de los efectos de las medidas se debe seguir el mismo proceso que hemos descrito hasta el momento, con un estudio de las presiones e impactos, y revisando todos los pasos del 1 al 9 de la tabla 1 . Los recursos humanos necesarios, las tipologías, los sistemas de referencia etc. deben ser contrastados a la luz de los nuevos conocimientos científicos, aunque después de la primera implementación el proceso no será nunca tan largo ni complejo.

\section{CONCLUSIONES}

La intensa actividad en el estudio y aplicación del biomonitoreo para su uso en la gestión en España ha dado lugar a múltiples trabajos, y seguramente seguirá dándolo durante mucho tiempo. De acuerdo con lo que se ha publicado en Limnetica, los avances que se han realizado en la aplicación de los índices, y más particularmente los relacionados con la aplicación de la DMA, se van incorporando a los estudios más recientes, pero todavía hay algunos que ignoran las pautas necesarias para que estos puedan ser contrastables. Para el futuro, sería importante que tanto en los trabajos que se publican, como en los informes que realiza la administración, se cumplie- ran los 10 puntos que hemos explicado en este trabajo (Tabla 2).

Utilizando el decálogo anterior es posible diseñar un sistema de evaluación del estado de salud de los ríos de una cuenca que posteriormente podrá ser comparado entre cuencas y diversas regiones. Este decálogo debería resolver todas las dudas de cómo realizar un proceso de diseño e implementación de una red de biomonitoreo que cumpla las especificaciones de la DMA. En este sentido, muy recientemente se ha realizado una evaluación sobre la situación del estado químico y ecológico de las masas de agua en España. Los resultados son muy claros, primero falta de información (43\% de las masas de agua sin información); más del $50 \%$ en mal estado; y no todos los indicadores han sido utilizados (Willaarts et al., 2103). A nivel europeo la situación no es muy diferente. El reciente informe de la Agencia Ambiental Europea (EEA, 2012), al respecto del estado de las masas de agua, y su futura proyección fruto del análisis de los Planes de Gestión o Planes Hidrológicos presentados por los diversos Estados miembros de la UE, muestra que tan solo un $53 \%$ de las masas de agua lograrán conseguir el buen estado a finales de 2015. Adicionalmente, la información proporcionada por los estados miembros al respecto del estado actual de las aguas superficiales y subterráneas es, en muchos casos, limitada. En el $50 \%$ de las masas de agua de transición, o entre el 30 y el $40 \%$ de las masas de agua ríos, lagos y aguas costeras, el estado de las masas de agua es aún desconocido.

Queda pues mucho trabajo por realizar, y lo importante es que se realice con métodos contrastados e intercalibrados. Dados los conocimientos que tenemos y los protocolos que existen, los autores proponemos unas pautas con la intención de ayudar a los que se interesen por los estudios de biomonitoreo, sobre todo a las administraciones gestoras y competentes en el mantenimiento y consecución del buen estado de las masas de agua.

Finalmente, ¿cuál es el futuro del biomonitoreo? Según Woodward et al. (2013) el proceso que hemos descrito aquí podría estar obsoleto en pocos años si la taxonomía molecular y la secuenciación de nueva generación (NGS) se imponen en los estudios de biomonitoreo. Ello 
implicaría trabajar con una lista mucho mayor de especies y aplicar otras técnicas de bioevaluación mucho más complejas que las métricas que utilizamos actualmente. Pero este es otro asunto que deberá ser abordado en el futuro cuando exista un número suficiente de trabajos sobre el tema.

\section{REFERENCIAS}

ALBA-TERCEDOR, J. \& A. SÁNCHEZ-ORTEGA. 1988. Un método rápido y simple para evaluar la calidad biológica de las aguas corrientes basado en el de Helawell (1978). Limnetica, 4: 51-56.

ALBA-TERCEDOR, J. \& N. PRAT. 1992. Spanish experience in the use of macroinvertebrates as biological pollution indicators. In: River Water Quality Assessmentand Control: 733-738. CCEE, Bruselas, Bélgica.

ALBA-TERCEDOR, J., P. JÁIMEZ-CUÉLLAR, M. ÁLVAREZ, J. AVILÉS, N. BONADA, J. CASAS, A. MELlADO, M. ORTEGA, I. PARDO, N. PRAT, M. RIERADEVALL, S. ROBLES, C. E. SÁINZ-CANTERO, A. SÁNCHEZ-ORTEGA, M. L. SUÁREZ, M. TORO, M. R. VIDAL-ALBARCA, S. VIVAS \& C. ZAMORA-MUÑOZ. 2002. Caracterización del estado ecológico de los ríos mediterráneos ibéricos mediante el índice IBMWP (antes $\mathrm{BMWP}^{\prime}$ ). Limnetica, 21(3-4): 175-185.

ARMITAGE, P. D., I. PARDO, M. T. FURSE, \& F. J. WRIGHT. 1990. Assessment and prediction of biological quality. A demonstration of a British macroinvertebrate-based method in two Spanish rivers. Limnetica, 6: 147-156.

BAILEY, R. C., M. G. KENNEDY, M. Z. DERVISH \& R. M. TAYLOR. 1998. Biological assessment of freshwater ecosystems using a reference condition approach: Comparing predicted and actual benthic invertebrate communities in Yukon streams. Freshwater Biology, 39(4): 765-774.

BARATA, C., C. PORTE \& D. J. BAIRD. 2004. Experimental designs to assess endocrine disrupting effects in Invertebrates, a review. Ecotoxicology, 13(6): 511-517.

BARBOUR, M. T., J. GERRITSEN, B. D.SNYDER \& J. B. STRIBLING. 1999. Rapid bioassessment protocols for use in streams and wadeable rivers: Periphyton, Benthic Macroinvertebrates and Fish. $2^{\text {nd }}$ edition, EPA841-B-99-002. U. S. Environmen- tal Protection Agency, Office of Water, Washington, D.C.

BARBOUR, M. T. J. B. STRIBLING, \& J. R. KARR. 1995. Multimetric approach for establishing biocriteria and measuring biological condition. In: Biological Assessment and Criteria. Tools for Water Resource Planning and Decision Making. W. S. Davis \& T.P. Simon (eds). Boca Raton-London-Tokyo, Lewis Publishers.

BARQUIN, J. \& F. MARTINEZ-CAPEL. 2011. Preface: Assessment of physical habitat characteristics in rivers, implications for river ecology and management. Limnetica, 30(2) 159-168.

BENEJAM, L., E. APARICIO, M. J. VARGAS, A. VILA-GISPERT \& E. GARCÍA-BERTHOU. 2008. Assessing fish metrics and biotic indices in a Mediterranean stream: Effects of uncertain native status of fish. Hydrobiologia, 603: 197-210.

BENEJAM, L., P. L.ANGERMEIER, A. MUNNÉ \& E. GARCÍA-BERTHOU. 2010. Assessing effects of water abstraction on fish assemblages in Mediterranean streams. Freshwater Biology, 55: 628642.

BIRK, S., W. BONNE, A. BORJA, S. BRUCET, A. COURRAT, S. POIKANE, A. SOLIMINI, W. VAN DE BUND, N. ZAMPOUKAS \& D. HERING. 2012. Three hundred ways to assess Europe's surface waters: An almost complete overview of biological methods to implement the Water Framework Directive. Ecological Indicators, 18: 31-41.

BONADA, N., N. PRAT, A. MUNNÉ, M. PLANS, C. SOLÀ, M. ÁLVAREZ, I. PARDO, G. MOYÀ, G. RAMON, M. TORO, S. ROBLES, J. AVILÉS, M. L. SUÁREZ, M. R. VIDAL-ABARCA, A. MELLADO, J. L. MORENO, C. GUERRERO, S. VIVAS, M. ORTEGA, J. CASAS, A. SÁNCHEZ-ORTEGA, P. JÁIMEZ-CUÉLLAR \& J. ALBA-TERCEDOR. 2002a. Ensayo de una tipología de las cuencas mediterráneas del proyecto Guadalmed siguiendo las directrices de la directiva marco del agua. Limnetica, 21(3-4), 77-98.

BONADA, N., N. PRAT, A. MUNNÉ, M. PLANS, C. SOLÀ, M. ÁLVAREZ, I. PARDO, G. MOYÀ, G. RAMON, M. TORO, S. ROBLES, J. AVILÉS, M. L. SUÁREZ, M. R. VIDAL-ABARCA, A. MELLADO, J. L. MORENO, C. GUERRERO, S. VIVAS, M. ORTEGA, J. CASAS, A. SÁNCHEZ-ORTEGA, P. JÁIMEZ-CUÉLLAR \& J. ALBA-TERCEDOR. 2002b. Intercalibración de la metodología Guadalmed. Selección de un protocolo de 
muestreo para la determinación del estado ecológico de los ríos mediterráneos. Limnetica, 21(3-4), 13-33.

BONADA, N., N. PRAT, A. MUNNÉ, M. RIERADEVALL, J. ALBA-TERCEDOR, M. ÁLVAREZ, J. AVILÉS, J. CASAS, P. JÁIMEZ-CUÉLLAR,A. MELLADO, G. MOYÀ, I. PARDO, S. ROBLES, G. RAMON, M. L. SUÁREZ, M. TORO, M. R. VIDAL-ABARCA, S. VIVAS \& C. ZAMORAMUÑOZ. 2002c. Criterios para la selección de condiciones de referencia en los ríos mediterráneos. Resultados del proyecto Guadalmed. Limnetica, 21(3-4), 99-114.

BONADA, N., N. PRAT, V. H. RESH, \& B. STATZNER. 2006. Developments in Aquatic Insect Biomonitoring: A comparative analysis of recent approaches. Annual Review of Entomology, 51: 495523.

BUFFAGNI, A. \& M.T. FURSE. 2006. Intercalibration and comparison -major results and conclusions from the STAR project. Hydrobiologia, 566: 357-364.

CHAVES, M., J. L. COSTA, P. CHAINHO, M. J. COSTA \& N. PRAT. 2006. Selection and validation of reference sites in small river basins. Hydrobiologia, 573(1): 133-154.

CHAVES, M., J. L. COSTA, P. CHAINHO, M. J. COSTA \& N. PRAT. 2011. Are Water Framework Directive stream types biologically relevant? The case of the Mondego River, Portugal. Annales de Limnologie-International Journal of Limnology, 47: 119-131.

CHOVAREC, A., P. JÄGER, M. JUNGWIRTH, V. KOLLER-KREIMEL, O. MOOG \& S. MUHAR. 2000. The Austrian way of assessing ecological integrity of running waters: A contribution to the EU Water Framework Directive. Hydrobiologia, 422/423: 445-452.

DAMASIO, J. B., C. BARATA, A. MUNNÉ, A. GINEBREDA, H. GUASCH, S. SABATER, J. CAIXACH \& C. PORTE. 2007. Comparing the response of biochemical indicators (biomarkers) and biological indices to diagnose the ecological impact of an oil spillage in a Mediterranean river (NE Catalunya, Spain). Chemosphere, 66: 1206-1216.

DESCY, J. P. 1979. A new approach to water quality estimation using diatoms. Nova Hedwigia 64: 305-323.

DOBERSTEIN, C. P., J. R.KARR, L. L. CONQUEST. 2000. The effect of fixed-count subsam- pling on macroinvertebrate biomonitoring in small streams. Freshwater Biology, 44: 355-371

DODKINS, I., B. RIPPEY, T.J. HARRINGTON, C. BRADLEY, B. N.CHATHAIN, M. KELLYQUINN, M. MCGARRIGLE, S. HODGE \& D. TRIGG. 2005. Developing an optimal river typology for biological elements within the Water Framework Directive. Water Research, 29: 3479_ 3486.

EUROPEAN COMMISSION. 2000. Directiva 2000/ 60/CE del Parlamento Europeo y el Consejo de 23 de octubre de 2000 por la que se establece un marco comunitario de actuación en el ámbito de la política de aguas. Diario Oficial de la Comunidad Europea. L 327 de 22 de Diciembre de 2000: 1-72.

EUROPEAN COMMISSION. 2005. Common Implementation Strategy for the Water Framework Directive (2000/60/EC)-Guidance Document No. 14. Guidance on the Intercalibration Process 20042006. EUROPEAN COMMISSION, 2007. MedGIGIntercalibration technical report-Part $1 \mathrm{Ri}-$ vers, Section 1 Benthic Invertebrates. 15 June 2007.

EUROPEAN COMMISSION. 2008. Establishing, pursuant to Directive 2000/60/EC of the European Parliament and of the Council, the values of the Member State monitoring system classifications as a result of the intercalibration exercise (Commission Decision 2008/915/EC). Official Journal of the European Union (10/12/2008), L 332: 20-44.

EUROPEAN COMMISSION. 2013. Establishing, pursuant to Directive 2000/60/EC of the European Parliament and of the Council, the values of the Member State monitoring system classifications as a result of the intercalibration exercise and repealing Decision 2008/915/EC. Commission Decision 2013/480/EU. Official Journal of European Union, L266/1. 47 pp.

EEA -EUROPEAN ENVIRONMENT AGENCY. 2012. European Waters: Assessment of Status and Pressures. EEA Report $n^{\circ}$ 8/2012. (http://www.eea. europa.eu/publications/european-waters-assessment-2012).

FAUSCH, K. D., J. R. KARR \& P. R. YANT. 1984. Regional application of an index of biotic integrity based on stream fish communities. Transactions of the American Fisheries Society, 113(1): 39-55.

FEIO, M. J., S. F. P. ALMEIDA, S. C. CRAVEIRO \& A. J. CALADO.2009. A comparison between biotic indices and predictive models in stream water 
quality assessment based on benthic diatom communities. Ecological Indicators, 9: 497-507.

FEIO, M. J. \& J. M. POQUET. 2011. Predictive models for freshwater biological assessment: Statistical approaches, biological elements and the Iberian Peninsula experience: A review. International Review of Hydrobiology, 96: 321-346.

FEIO, M. J., F. C. AGUIAR, S. F. P.ALMEIDA, J. FERREIRA, M. T. FERREIRA, C. ELIAS, S. R. S. SERRA, A. BUFFAGNI, J. CAMBRA, C. CHAUVIN, F. DELMAS, G. DÖRFLINGER, S. ERBA, N. FLOR, M. FERRÉOL, M. GERM, L. MANCINI, P. MANOLAKI, S. MARCHEGGIANI, M.R. MINCIARDI, A. MUNNÉ, E. PAPASTERGIADOU, N. PRAT, C. PUCCINELLI, J. ROSEBERY, S. SABATER, S. CIADAMIDARO, E. TORNÉS, TZIORTZISI, G. URBANIČ \& C. VIEIRA. 2013. Least disturbed conditions for European Mediterranean rivers. Science of Total Environment, http://dx.doi.org/10.1016/j.scitotenv.2013.05.056

FERREIRA, M. T., P. M. RODRÍGUEZ-GONZÁLEZ, F. C. AGUIAR \& A. ALBUQUERQUE. 2005. Assessing biotic integrity in Iberian rivers: Development of a multimetric plant index. Ecological Indicators, 5: 137-149.

FERRÉOL, M., A. DOHET, H. M. CAUCHIE \& L. HOFFMANN. 2005. A top-down approach for the development of a stream typology based on abiotic variables. Hidrobiologia, 551: 193-208.

FRIBERG, N., L. SANDIN, M. T.FURSE, S. E. LARSEN, R. T. CLARKE \& P. HAASE. 2006. Comparison of macroinvertebrate sampling methods in Europe. Hydrobiology, 566: 365-378.

FRISSELL, C. A., W. J. LISS, C. E. WARREN \& M. D. HURREY. 1986. A hierarchical framework for stream habitat classification: Viewing streams in a watershed context. Environmental Management, 10 (2): 199-214.

GERHARDT, A. 1999. Biomonitoring for the 21st. century. Environmental Research Forum, 9: 1-12.

GONZÁLEZ DEL TÁNAGO, M. \& D. GARCÍA DE JALÓN. 1984. Desarrollo de un índice biológico para estimar la calidad de las aguas de la Cuenca del Duero. Limnetica, 1: 263-272.

HAURY, J., M. C. PELTRE, M. TRÉMOLIÈRES, J. BARBE, G. THIÉBAUT, J. BERNEZ, H. DANIEL, P. CHATENET, G. HAAN-ARCHIPOF, S. MULLER, A. DUTARTRE, C. LAPLACETREYTURE, A. CAZAUBON \& E. LAMBERTSERVIEN. 2006. A new method to assess water trophy and organic pollution-The Macrophyte
Biological Index for Rivers (IBMR): Its application to different types of river and pollution. In: Macrophytes in Aquatic Ecosystems: From Biology to Management Developments, 190: 153-158. HEISKANEN, A. S., W. VAN DE BUND, A. C. CARDOSO \& P. NOGES. 2004. Towards good of ecological status of surface waters in EuropeInterpretation and harmonisation of the concept. Water Science and Technology, 49: 169-17.

HELLAWELL, J. M. 1986. Biological Indicators of Freshwater Pollution and Environmental Management. K. Mellanby, Ed. Elsevier Applied Science. London \& New York.

HERING, D., A. BUFFAGNI, O. MOOG, L. SANDIN, M. SOMMERHÄUSER, I. STUBAUER, C. FELD, R. JOHNSON, P. PINTO, N. SKOULIKIDIS, P. VERDONSCHOT \& S. ZAHRÁDKOVÁ. 2003. The development of a system to assess the ecological quality of streams based on macroinvertebrates-Design of the sampling programme within the AQEM Project. International Review of Hydrobiology, 88: 345-361.

ILLIES, J. \& L. BOTOSANEANU. 1963. Problemes et methodes de la classification et de la zonation ecologique des eaux corantes, considereés surtout du point de vue faunistique. Mitteilungen der Internationale Vereinigungfür Theoretische and Angewandte Limnologie 12: 1-57.

JAIMEZ-CUÉLLAR, P., S. VIVAS, N. BONADA, S. ROBLES, A. MELLADO, M. ÁLVAREZ, J. AVILÉS, J. CASAS, M. ORTEGA, I. PARDO, N. PRAT, M. RIERADEVALL, C. SÁINZ-CANTERO, A. SÁNCHEZ-ORTEGA, M. L. SUÁREZ, M. TORO, M. R. VIDAL-ABARCA, C. ZAMORA-MUÑOZ \& J. ALBA-TERCEDOR. 2002. Protocolo Guadalmed (PRECE). Limnetica, 21(3-4): 187-204.

KARR, J. R., \& E. W. CHU. 1999. Restoring Life in Running Waters: Better Biological Monitoring. Island Press, Washington, D. C.

KELLY, M. G., C. J. PENNY \& B. A. WHITTON.1995. Comparative performance of benthic diatom indices used to assess river water quality. Hydrobiologia, 302: 179-188.

LEEKS, G. J. L., C. NEALA, H. P. JARVIE, H. CASEY \& D. V. LEACH. 1997. The LOIS river monitoring network: Strategy and implementation. Science of the Total Environment, 194-195: 101-109.

MARGALEF, R. 1969. El concepto de polución y sus indicadores biológicos. Documentos de investiga- 
ción hidrológica. Suplemento de la revista Agua, 7: 105-133.

MARM - MINISTERIO DE MEDIO AMBIENTE MEDIO RURAL Y MARINO. 2008. ORDEN ARM/2656/2008, de 10 de septiembre, por la que se aprueba la instrucción de planificación hidrológica (IPH). Boletín Oficial del Estado-BOE, 229: 38472-38582.

MINSHALL, G. W., K. W. CUMMINS, R. C. PETERSEN, C. E. CUSHING, D. A. BRUNS, J. R. SEDELL \& R. L. VANNOTE. 1985. Developments in stream ecosystem theory. Canadian Journal of Fisheries and Aquatic Sciences, 42: 10451055.

MONDY, C. P., B. VILLENEUVE, V. ARCHAIMBAULT \& P. USSEGLIO-POLATERA. 2012. A new macroinvertebrate-based multimetric index (I2M2) to evaluate ecological quality of French wadeable streams fulfilling the WFD demands: A taxonomical and trait approach. Ecological Indicators, 18: 452-467.

MORENO, J. L., C. NAVARRO, J. DE LAS HERAS. 2006. Propuesta de un índice de vegetación acuática (IVAM) para la evaluación del estado trófico de los ríos de Castilla-La Mancha: Comparación con otros índices bióticos. Limnetica, 25(3): 821-838.

MUNNÉ, A. \& N. PRAT. 1998. Delimitación de regiones ecológicas en la cuenca del Ebro. Confederación Hidrográfica del Ebro, Zaragoza.

MUNNÉ, A. \& N. PRAT. 2002. Desarrollo de herramientas de análisis y tipificación ecológica de los sistemas fluviales en las cuencas internas de Cataluña. In: La directiva marco: Realidades y futuros. III Congreso Ibérico sobre Gestión y Planificación del Agua. Sevilla, Noviembre 2002.

MUNNÉ, A., N. PRAT, C. SOLÀ, N. BONADA \& M. RIERADEVALL. 2003. A simple field method for assessing the ecological quality of riparian habitat in rivers and streams: QBR index. Aquatic Conservation-Marine and Freshwater Ecosystems, 13: 147-163.

MUNNÉ, A. \& N. PRAT. 2004. Defining river types in a Mediterranean area. A methodology for the implementation of the EU Water Framework Directive. Environmental Management, 34(5): 711729.

MUNNÉ, A. \& N. PRAT. 2009. Use of macroinvertebrate-based multimetric indices for water quality evaluation in Spanish Mediterranean rivers: An intercalibration approach with the IBMWP index. Hydrobiologia, 628: 203-205.
MUNNÉ, A. \& N. PRAT. 2011. Effects of Mediterranean climate annual variability on stream biological quality assessment using macroinvertebrate communities. Ecological Indicators, 11(2): 651662.

MUNNÉ, A., L. TIRAPU, C. SOLÀ, L. OLIVELLA, M. VILANOVA, A. GINEBREDA \& N. PRAT. 2012. Comparing chemical and ecological status in Catalan rivers. Analysis of river quality status following the Water Framework Directive. In: Emerging and Priority Pollutants in Rivers: Bringing Science into River Management Plans. H. Guasch et al. (eds.). The Handbook of Environmental Chemistry, 19: 243-266. Springer-Verlag Berlin Heidelberg.

NEWBOLD, C. \& N. T. H. HOLMES. 1987. Nature conservation: Water quality criteria and plants as water quality monitors. Water Pollution Control, 86: 345-364.

NIJBOER, R. C., R. K. JOHNSON, P. F. M. VERDONSCHOT, M. SOMMERHÄ USER \& A. BUFFAGNI. 2004. Establishing reference conditions for European streams. Hydrobiologia, 516: 91-105.

OMERNIK, J. M. \& R. G. BAILEY. 1997. Distinguishing between watersheds and ecoregions. Journal of the American Water Resources Association, 33(5): 935-949.

OSCOZ, J., J. GOMÀ, L. ECTOR, J. CAMBRA, M. PARDOS, C. DURÁN. 2007. Estudio comparativo del estado ecológico de los ríos de la cuenca del Ebro mediante macroinvertebrados y diatomeas. Limnetica, 26(1): 143-158.

PETTS, G. E. 2008. Hydroecology: The scientific basis for water resources management and river regulation. In: Hydroecology and Ecohydrology: Past, Present and Future. P. J. Wood, D. M. Hannah \& J. P. Sadler (eds), John Wiley \& Sons, Ltd, Chichester, UK. Doi: 10.1002/9780470010198.ch13.

POFF, N. L \& H. K. H. ZIMMERMAN.2010. Ecological responses to altered flow regimes: A literature review to inform the science and management of environmental flows. Freshwater Biology, 55: 194-205.

PONT, D., B. HUGUENY \& C. ROGERS. 2007. Development of a fish-based index for the assessment of river health in Europe: The European Fish Index. Fisheries Management and Ecology, 14: 427439.

POQUET, J. M., J. ALBA-TERCEDOR, T. PUNTÍ, M. M. SÁNCHEZ-MONTOYA, S. ROBLES, M. 
ÁLVAREZ, C. ZAMORA-MUÑOZ, C. E. SÁINZCANTERO, M. R. VIDAL-ABARCA, M. L. SUÁREZ, M. TORO, A. M. PUJANTE, M. RIERADEVALL \& N. PRAT. 2009. The MEDiterranean Prediction and Classification System (MEDPACS): An implementation of the RIVPACS/AUSRIVAS predictive approach for assessing Mediterranean aquatic macroinvertebrate communities. $\mathrm{Hy}$ drobiologia, 623: 153-171.

PRAT, N., M. A. PUIG, G. GONZÁLEZ \& L. TORT. 1983. Predicció $i$ control de la qualitat de les aigües dels rius Besòs i Llobregat. II: El poblament faunístic i la seva relació amb la qualitat de les aigües. Monografies, Diputació de Barcelona. Servei del Medi Ambient. Barcelona

PRAT, N. 2002. El proyecto GUADALMED. Limnetica, 21(3-4): 1-4.

PRAT, N., P. FORTUÑO \& M. RIERADEVALL. 2009. Manual d'utilització de l'Índex d'Hàbitat Fluvial (IHF). Diputació de Barcelona. DL: B-37099-2009.Barcelona.

PRAT, N., M. RIERADEVALL, C. BARATA \& A. MUNNÉ. 2013. The combined use of metrics of biological quality and biomarkers to detect the effects of reclaimed water on macroinvertebrate assemblages in the lower part of a polluted Mediterranean river (Llobregat River, NE Spain). Ecological Indicators, 24: 167-176.

PRYGIEL, J., M. COSTE, \& J. BUKOWSKA. 1999. Review of the major diatom-based techniques for the water quality assessment of rivers-state of the art in Europe. In: Use of Algae for Monitoring Rivers III. Prygiel J, B. A. Whitton \& J. Bukowska (eds). Agence de l'Eau Artois-Picardie, Douai, France.

QUIRÓS, L., B. PIÑA, M. SOLÉ, J. BLASCO, M. A. LÓPEZ, M. C. RIVA, D. BARCELÓ \& D. RALDÚA. 2007. Environmental monitoring by gene expression biomarkers in Barbus graellsii: Laboratory and field studies. Chemosphere, 67 (6): 1144 1154.

ROSENBERG, D. M. \& V. H. RESH. 1993. Introduction to freshwater biomonitoring and benthic macroinvertebrates. In: Freshwater Biomonitoring and Benthic Macroinvertebrates. D. M. Rosenberg \& V. H. Resh (eds.): Chapman \& Hall, New York.

SABATER, S., H. GUASCH, A. PICÓN, A. ROMANÍ \& I. MUÑOZ. 1996. Using diatom communities to monitor water quality in a river after the implementation of a sanitation plan (River Ter,
Spain). Use of Algae for Monitoring Rivers II. Whitton, B. A. \& E. Rott (eds).

SABATER, S. 2000. Diatom communities as indicators of environmental stress in the Guadiamar River, S-W. Spain, following a major mine tailings spill. Journal of Applied Phycology, 12(2): 113124.

SÁNCHEZ-MONTOYA, M. M., T. PUNTÍ, M. L. SUÁREZ, M. R. VIDAL-ABARCA, M. RIERADEVALL, J. M. POQUET, C. ZAMORAMUÑOZ, S. ROBLES, M. ÁlVAREZ, J. ALBATERCEDOR, M. TORO, A. M. PUJANTE, A. MUNNÉ \& N. PRAT. 2007. Concordance between ecotypes and macroinvertebrate assemblages in Mediterranean streams. Freshwater Biology 52(11), 2240-2255.

SÁNCHEZ-MONTOYA, M. M., M. R. VIDALABARCA, T. PUNTÍ, J. M. POQUET, N. PRAT, M. RIERADEVALL, J. ALBA-TERCEDOR, C. ZAMORA-MUÑOZ, M. TORO, S. ROBLES, M. ÁLVAREZ \& M. L. SUÁREZ. 2009. Defining criteria to select reference sites in Mediterranean stream. Hydrobiologia, 619:39-54.

SOSTOA, A., N. CAIOLA \& F. CASALS. 2004. A New IBI (IBICAT) for Local Application of the E.U. Water Framework Directive. 5th Ecohydraulics conference. Madrid, septiembre 2004.

SPONSELLER, R. A., E. F. BENFIELD \& H. M. VALETT. 2001. Relationships between land use, spatial scale and stream macroinvertebrate communities. Freshwater Biology, 46: 1409-1424.

STARK, J. D. 1993. Performance of the Macroinvertebrate Community Index: Effects of sampling method, sample replication, water depth, current velocity, and substratum on index values. New Zealand Journal of Marine and Freshwater Research, 27(4): 463-478.

SUÁREZ, M. L., A. MELLADO, M. M. SÁNCHEZMONTOYA \& M. R. VIDAL-ALBARCA. 2005. Propuesta de un índice de macrófitos (IM) para evaluar la calidad ecológica de los ríos de la cuenca del Segura. Limnetica, 24: 305-318.

TINER, R. W. 2004. Remotely-sensed indicators for monitoring the general condition of "natural habitat" in watersheds: An application for Delaware's Nanticoke River watershed. Ecological Indicators, 4(4): 227-243.

VILA-GISPERT, A., E. GARCÍA-BERTHOU \& R. MORENO-AMICH. 2002. Fish zonation in a Mediterranean stream: Effects of human disturbances. 
Aquatic Sciences - Research Across Boundaries, 64(2): 163-170.

VINSON, M. R. \& C. P. HAWKINS. 1998. Biodiversity of stream insects: Variation at local, basin, and regional scales. Annual Review of Entomology: 43: 271-293.

WALLIN, M., T. WIEDERHOLM \& R. JOHNSON. 2003. Guidance on establishing reference conditions and ecological status class boundaries for inland surface waters. Common Implementation Strategy Working Group 2.3-REFCOND Guidance. Final version 7.0.

WARRY, N. D. \& M. HANAU. 1993. The use of terrestrial Ecoregions as regional-scale screen for selecting representative reference sites for water quality monitoring. Environmental Management, 17(2): 267-276.

WILLAARTS, B. A., M. BALLESTEROS \& N. HERNÁNDEZ-MORA. 2013. Ten years of the
Water Framework Directive in Spain: An overview of the ecological and chemical status of surface water bodies. In: "Integrated Water Resources Management in the XXIst Century", VI Botín Foundation Water Workshop. P. Martínez Santos \& M. Aldaya (eds.) Taylor \& Francis Group.

WOODIWISS, F. S. 1964. The biological system of stream classifications used by the Trand River Board. Chemyistry and Industry, 11: 443-447.

WOODWARD, G., C. GRAY \& D. J. BAIRD. 2013. Biomonitoring for the 21 st century: New perspectives in an age of globalisation and emerging environmental threats. Limnetica, 32(2): 159-174.

WALLIN, M., T. WIEDERHOLM \& R. K. JOHNSON. 2003. Guidance on establishing reference conditions and ecological status class boundaries for inland surface waters Produced by CIS Working Group 2.3-REFCOND Guidance. European Commission. 